\title{
RESPUESTA
}

\section{MODELOS FARMACOECONÓMICOSDE SIMULACIÓN, METAANÁLISIS Y PATROCINIO DE LA INDUSTRIA}

Inmaculada Gómez Arrayás (1), Carmen Suárez Fernández (2), Jorge F Gómez Cerezo (3), Lourdes Betegón Nicolás (4), Marina de Salas-Cansado (5) y Carlos Rubio-Terrés (6).

(1) Hospital Ruber Internacional. Madrid.

(2) Hospital Universitario de La Princesa. Madrid.

(3) Hospital Universitario Infanta Sofía. Madrid.

(4) Bristol-Myers Squibb España. Madrid.

(5) Pfizer España. Madrid.

(6) Health Value. Madrid.

\begin{abstract}
Agradecemos el interés mostrado en nuestro artículo ${ }^{1}$. El autor de la carta comenta dos aspectos: en primer lugar, la validez de un metaanálisis en red (network meta-analysis) para efectuar comparaciones indirectas. En segundo lugar, expresa algunas opiniones personales sobre la objetividad y validez de los estudios patrocinados por la industria farmacéutica.
\end{abstract}

Cuando no se dispone de una comparación directa entre dos tratamientos relevantes de una enfermedad, lo que procede es realizar un ensayo clínico aleatorizado. Si el ensayo clínico no puede efectuarse, los investigadores pueden recurrir a una comparación indirecta de los tratamientos mediante metaanálisis en red e interpretar los resultados, con precaución ${ }^{2}$. En este sentido, la utilidad (y también las limitaciones) de este tipo de metaanálisis son reconocidas por la inmensa mayoría de las agencias evaluadoras, como la Canadian

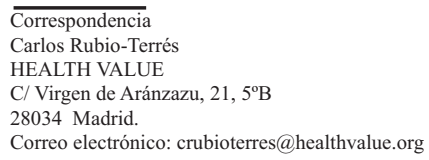

Agency for Drugs and Technologies in Health (CADTH) ${ }^{2}$, el National Institute for Health and Clinical Excellence $(\mathrm{NICE})^{3}$ o la propia Agencia Española de Medicamentos y Productos Sanitarios ${ }^{4}$.

Respecto al metaanálisis en red en el que se basa nuestro estudio, su metodología no se ha reproducido en nuestro artículo de manera exhaustiva, aunque sí suficientemente, dado que ésta se explica extensamente en la publicación original del metaanálisis en la revista Clinical and Applied Thrombosis/Hemostasis ${ }^{5}$ que es revisada por pares. Las limitaciones de este metaanálisis son claramente descritas en esa publicación. La explicación de las inconsistencias observadas entre algunos resultados de las comparaciones directas y las indirectas está publicada en el informe del Evidence Review Group ${ }^{6}$. A este respecto, es de interés señalar que un metaanálisis de comparaciones indirectas publicado recientemente por investigadores de la Agencia Española de Medicamentos y Productos Sanitarios ${ }^{4}$ concluye que, en comparación con enoxaparina, el riesgo relativo de hemorragias clínicamente relevantes fue menor con apixaban $(0,82$; 
IC95\% 0,69 a 0,$98 ; p=0,03$ ) pero no con dabigatrán y rivaroxaban. En este metaanálisis no se hace referencia a inconsistencias entre comparaciones directas e indirectas ${ }^{4}$. Según otros dos recientes metaanálisis, apixaban parece estar asociado a un menor riesgo de hemorragias en comparación con rivaroxaban ${ }^{7} \mathrm{y}$ enoxaparina ${ }^{8}$. Así mismo, en un metaanálisis realizado en Estados Unidos se concluyó que apixaban es más efectivo que enoxaparina sin aumentar el riesgo de sangrados ${ }^{9}$.

En nuestro artículo, revisado por pares, se indican claramente los puntos débiles del estudio, indicando que "debe recordarse que se trata de un modelo teórico que, por definición, es una simulación simplificada de la realidad" y que, así mismo, "debe tenerse en cuenta [...] que la población estudiada en los ensayos clínicos fue seleccionada en base a unos criterios de inclusión y, por tanto, no refleja a la población real atendida en la práctica cotidiana, con comorbilidades importantes, como la insuficiencia renal"1.

En cuanto al segundo aspecto comentado por el autor de la carta, sobre la objetividad y validez de los estudios patrocinados por la industria farmacéutica, queremos hacer varias consideraciones. La validez de una investigación debe valorarse única y exclusivamente mediante la crítica objetiva de los métodos empleados. La descalificación de un estudio por el simple hecho de su patrocinio no nos parece científica sino fruto de una visión sesgada y de prejuicios que nada tienen que ver con el método científico. El autor de la carta dice que "los estudios sobre fármacos promovidos por la industria deberían ser leídos con cautela". ¿Y por qué no los no promovidos por la industria? A nuestro juicio toda la investigación, independientemente de quién la patrocine, debe ser objeto de escrutinio científico ("el interés directo en los resultados" de un estudio puede estar determinado no solamente por el patrocinio, sino también por intereses políticos, ideológicos, morales o personales). De lo contrario, estaríamos ante prejuicios que -como decíamos antes- nada tienen que ver con la calidad de las investigaciones sanitarias, siendo meras opiniones subjetivas sin ningún valor en el contexto de la medicina basada en las pruebas. Por el contrario, poner en duda la excelencia científica o la honorabilidad de aquellos investigadores que trabajan en o para la industria farmacéutica llevaría al absurdo de invalidar la inmensa mayoría de la investigación clínica realizada en los últimos decenios. Los investigadores del Sistema Nacional de Salud (SNS) colaboran habitualmente en estudios patrocinados por la industria farmacéutica. La puerta a investigaciones clínicas patrocinadas por el propio SNS está expedita, pero lamentablemente pocas veces se traspasa ese umbral. La crítica de la metodología de un estudio o investigación es absolutamente conveniente y necesaria. La descalificación global de los investigadores que colaboran en estudios patrocinados por la industria farmacéutica no es aceptable. Nadie debe poner en duda que ningún interés secundario deba prevalecer sobre el principal de la investigación sanitaria: la salud de los enfermos y la integridad de la investigación ${ }^{10}$.

\section{BIBLIOGRAFÍA}

1.Gómez I, Suárez C, Gómez JF, Betegón L, de SalasCansado M, Rubio-Terrés C. Impacto presupuestario para el Sistema Nacional de Salud de la prevención del tromboembolismo venoso con apixaban en pacientes sometidos a artroplastia total de rodilla o cadera. Rev Esp Salud Pública. 2012; 86: 591-602. Wells

2.Wells GA, Sultan SA, Chen L, Khan M, Coyle D. Indirect Evidence: Indirect Treatment Comparisons in Meta-Analysis. Ottawa: Canadian Agency for Drugs and Technologies in Health; 2009.

3.NICE. Decision Support Unit. Evidence synthesis TSD series. Disponible: http://www.nicedsu.org.uk/EvidenceSynthesis-TSD-series\%282391675\%29.htm (consulta: enero de 2013) 
4.Gómez-Outes A, Terleira-Fernández A, SuárezGea L, Vargas-Castrillón E. Dabigatran, rivaroxaban, or apixaban versusenoxaparin for thromboprophylaxis after total hip or knee replacement: systematic review, meta-analysis, and indirect treatment comparisons. BMJ. 2012 Jun 14;344:e3675. doi: 10.1136/bmj.e3675.

5.Cohen A, Drost P, Marchant N, Mitchell S, Orme M, Rublee D, Simon TA, Sutton A. The efficacy and safety of pharmacological prophylaxis of venous thromboembolism following elective knee or hip replacement: systematic review and network metaanalysis. Clin Appl Thromb Hemost. 2012; 18: 61127.

6.Riemsma R, Joore M, Grutters J, Armstrong N, Misso K, Noake C, Tushabe DA, Deshpande S, Severens JL, Kleijnen J. Apixaban for the prevention of venous thromboembolism in people undergoing elective knee and hip replacement surgery: a Single Technology Appraisal. York: Kleijnen Systematic Reviews Ltd; 2011.

7.Alves C, Batel-Marques F, Macedo AF. Apixaban and rivaroxaban safety after hip and knee arthroplasty: a meta-analysis. J Cardiovasc Pharmacol Ther. 2012; 17: 266-76.

8.Huang J, Cao Y, Liao C, Wu L, Gao F. Apixaban versus enoxaparin in patients with total knee arthroplasty. A meta-analysis of randomised trials. Thromb Haemost. 2011; 105: 245-53.

9.Raskob GE, Gallus AS, Pineo GF, Chen D, Ramirez LM, Wright RT, Lassen MR. Apixaban versus enoxaparin for thromboprophylaxis after hip or knee replacement: pooled analysis of major venous thromboembolism and bleeding in 8464 patients from the ADVANCE-2 and ADVANCE-3 trials. J Bone Joint Surg Br. 2012; 94: 257-64.

10.Camí J. Conflicto de intereses e investigación clínica. Med Clin (Barc). 1995; 105: 174-9. 